\title{
Three-dimensional computed tomography angiography of the pulmonary veins and their anatomical variations: involvement in video-assisted thoracoscopic surgery-lobectomy for lung cancer
}

\author{
A. Fourdrain, F. De Dominicis, M. Bensussan, J. Iquille, S. Lafitte, D. Michel, P. Berna \\ Department of Thoracic Surgery, Amiens University Hospital, University of Picardy, France
}

[Received: 1 September 2016; Accepted: 7 November 2016]

Background: Identification and section of pulmonary veins are an essential part of anatomical pulmonary resections. Intraoperative misunderstandings of pulmonary venous anatomy can lead to serious complications such as bleeding and delayed lung infarction or necrosis. We evaluated principally the rate of pulmonary venous anatomical variations, and secondarily the reliability and clinical outcomes of a preoperative morphological analysis.

Materials and methods: Between November 2012 and October 2013, we studied 100 consecutive patients with highly suspected or diagnosed stage I-II primitive lung cancer lesion. The surgical procedure initially retained was video-assisted thoracoscopic surgery (VATS) pulmonary resections and we studied preoperatively the proximal pulmonary venous anatomy using 64 channels multi-detector computed tomography (CT)-scan angiography to describe the venous anatomical variations.

Results: There were 65 men and 35 women with a mean age of 63 years. A pulmonary venous anatomical variation was present in 36 (36\%) patients, and right-sided anatomical variations were more frequent than on left-sided ones $(25 \%$ vs. $11 \%)$. The most frequent variation encountered on the right side was the existence of three separate pulmonary veins (16\%), and on the left side a single pulmonary vein (8\%). Surgical conversion occurred in $21 \%$ and we didn't experience a pulmonary venous lesion $(0 \%)$ or a post-operative lung infarction ( $0 \%)$.

Conclusions: We described pulmonary venous anatomical variations and their frequency. Anatomical variations exist and preoperative assessment of pulmonary venous anatomy using CT scan is a useful tool in VATS lobectomy to avoid unnecessary extension of pulmonary resections or iatrogenic complications in lung cancer surgery. (Folia Morphol 2017; 76, 3: 388-393)

Key words: pulmonary vein, anatomical variation, lobectomy, lung cancer, left atrium, video-assisted thoracoscopic surgery (VATS)

Address for correspondence: Dr. A. Fourdrain, Department of Thoracic Surgery - Amiens University Hospital, University of Picardy, F-80054 Amiens Cedex 1 France, Laboratoire d'Anatomie et d'Organogénèse - University of Picardy, F-80036 Amiens Cedex 1 France, e-mail: fourdrain.alex@chu-amiens.fr 


\section{INTRODUCTION}

Existence of pulmonary vessel anatomical variations remains crucial in non-small cell lung cancer surgery. A thorough evaluation of lung vascularisation becomes more and more frequent in the preoperative evaluation for lung cancer when a fully video-assisted thoracoscopic surgery (VATS) is indicated. This evaluation was performed essentially for pulmonary arterial vessels [3]. Ligation of an arterial branch has not serious consequences over lung parenchyma (except in gases exchange percentage). However, a venous ligation of a non-resected segment or lobe might cause infarction and necrosis of the remaining lung parenchyma. During embryological development, the pulmonary veins (PVs) develop independently from the cardiac formation. These PVs are not part of the primary venous system (cardinal, umbilical and vitelline). Different theories exist to explain the PVs origin: extrusion from the left atrium and joining the pulmonary plexus and/or development of the PVs in the posterior mesocardium and secondary incorporation to the left atrium $[19,20]$. Late studies show that the primitive PV develops as an independent structure in the dorsal mesocardium and connects both to the pulmonary venous plexus and primitive atrium [2]. Anomalies in the incorporation of the common PV into the left atrium are responsible for different pulmonary venous anatomical variations. Regarding the number of PVs at the veno-atrial junction, a common right or left venous trunk or supernumerary PVs can exist [2]. A precise anatomical evaluation of PVs by lower dose radiation and high-performance computed tomography (CT)-scan is possible. This examination is useful in morphological evaluation of veno-atrial relation before VATS lobectomy, allowing an easier recognition and identification of the vessels involved. This study was performed to assess the lung venous anatomical variations, the primary endpoint was the number of pulmonary venous trunks on each side and our second objective was to evaluate the clinical and surgical outcome of these variations in VATS lobectomy.

\section{MATERIALS AND METHODS}

\section{Patients}

We studied 100 patients included prospectively with highly suspected or diagnosed stage I-II primitive lung cancer lesion in our Department of Thoracic Surgery over a 12 months period (November 2012 - October 2013). The surgical exploration or procedure was retained after a multidisciplinary concertation and VATS lobectomy was performed for known primitive lung cancer patient and for patient with positive frozen section for primitive lung cancer. After signed informed consent, patients were included in our Thoracic Department Database, with the serial DRCI T38 under the prior authorisation of our University Hospital review board.

\section{Procedure}

Each patient had a pulmonary venous anatomical assessment with a reproducible technique of lung vessels angiography using a 64 channel, contrast-enhanced, multidetector CT (GE Healthcare, Waukesha, WI, USA), performed in accordance with our centre's standardised procedure. The contrast material (IOMERON 350, Bracco Imaging, France) was injected at a rate of $1.5 \mathrm{~mL} / \mathrm{s}$ (total volume: $100 \mathrm{~mL}$ ) using a mechanical injector. The slice thickness was $0.6 \mathrm{~mm}$. The reconstruction was performed with a $0.6 \mathrm{~mm}$ step $70 \mathrm{~s}$ after injection of the contrast material. All data were reviewed by two experienced operators (a thoracic surgeon and a radiologist). The extra pericardial PVs were analysed in mediastinal window using a multiplanar mode and we used 3-dimensional reconstructions to confirm the anatomical findings. Preoperative findings were compared to intraoperative findings for the operated side.

\section{RESULTS}

\section{Patients}

Among the 100 patients included, 65 were men and 35 women, with a mean age of 63 (34-81) years and all patients have been evaluated radiologically preoperatively.

\section{Venous anatomical variations of the lung}

We did not find a patient with a bilateral pulmonary venous anatomical variation, all variations were unilateral. Among the 100 patients, we found 36 cases of anatomical variations regarding the number of veins joining the atrium (36\%) and right-sided variations were more frequent than on the left side ( $25 \%$ vs. $11 \%)$. These results are summarised in Figure 1. On the right side: the most common pattern was the existence of $2(75 \%)$ PVs. The middle lobe vein drained in the right upper PV but in 5 cases it drained in the right inferior PV (5\%). The most frequent anatomical variation we observed was the presence of 3 PVs with 3 separated ostia in the left atrium, one for each lobe (Fig. 2A, B), and this was assessed in $16(16 \%)$ patients. Also, 6 patients had 3 separate veins with a right superior $\mathrm{PV}$ receiving the middle lobe vein and the 2 others branches were one from superior segment of the right inferior lobe and the other drained the basilar segments (6\%) (Fig. 2C). In 1 patient there were 4 PVs; 1 right superior pulmonary vein, 1 middle lobe vein and 


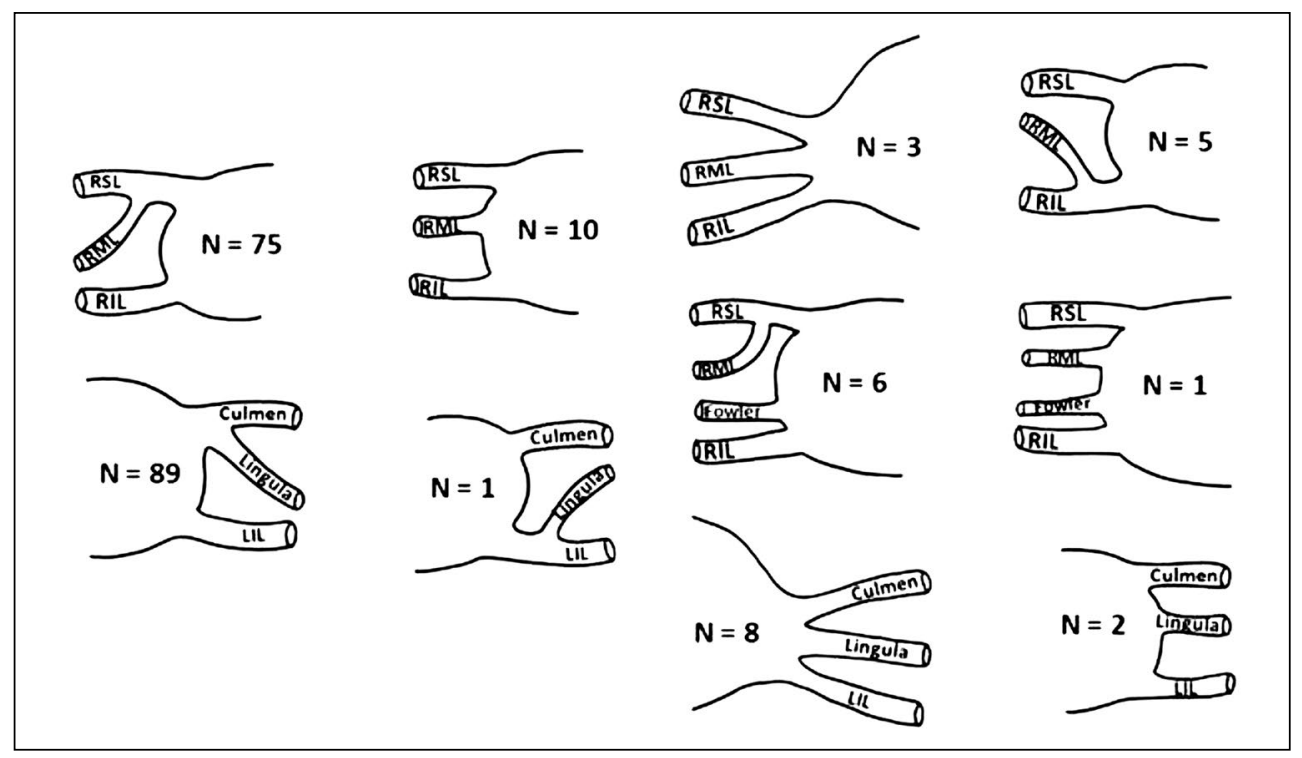

Figure 1. Distribution of the different pulmonary venous anatomical variations; Culmen — superior segment of left upper lobe pulmonary vein; Fowler superior segment of right lower lobe pulmonary vein; LIL — left inferior lobe pulmonary vein; Lingula — inferior segment of left upper lobe pulmonary vein; RIL — right inferior lobe pulmonary vein; RML — right middle lobe pulmonary vein; $\mathrm{RSL}$ — right superior lobe pulmonary vein.
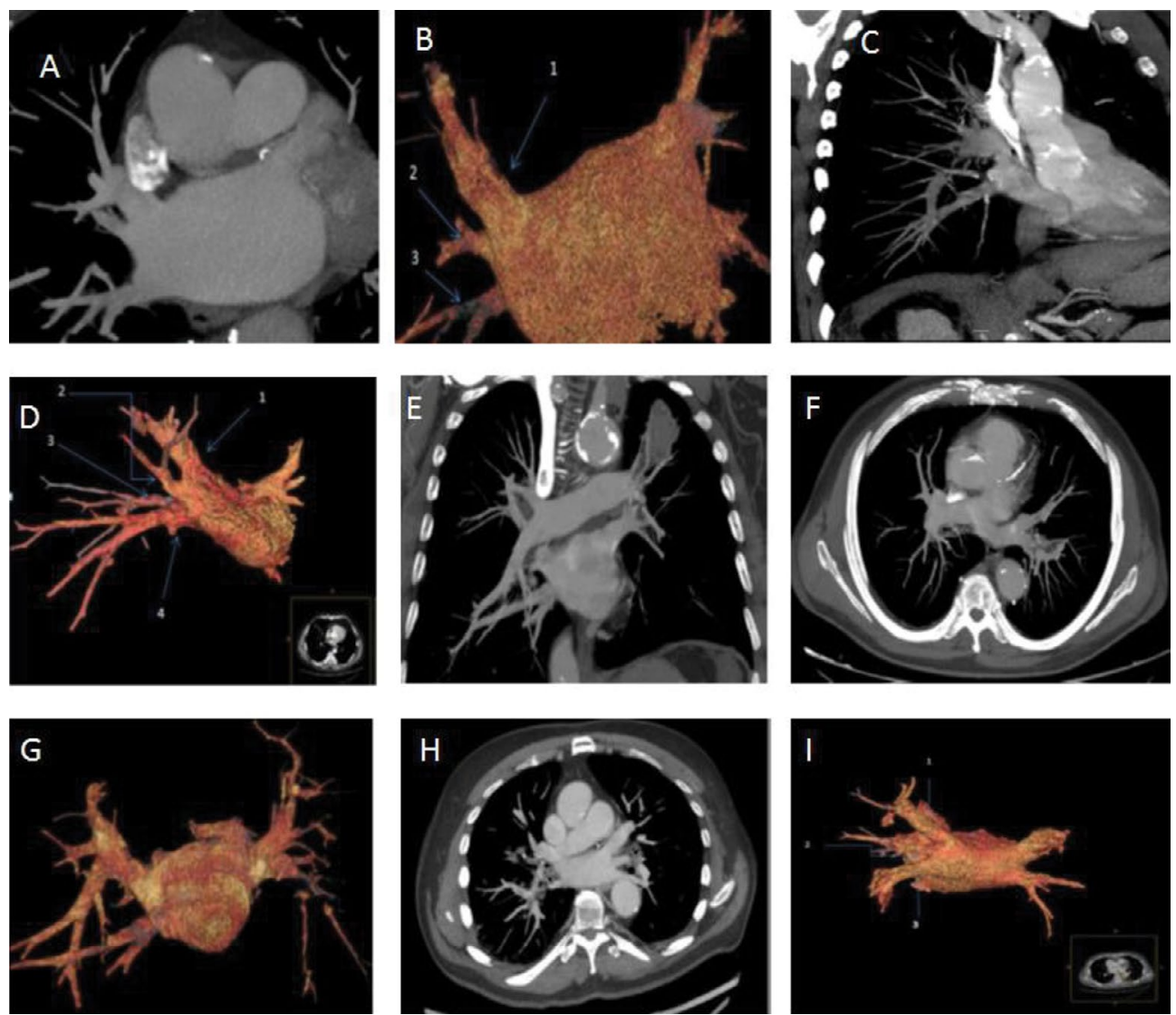

Figure 2. A. Computed tomography (CT) scan showing 3 right pulmonary veins; B. Three-dimensional (3D) reconstruction; 1 - right upper pulmonary vein; 2 - middle pulmonary vein; 3 - right lower pulmonary vein; C. CT scan showing a separated vein for the right upper segment of the lower lobe; $\mathbf{D}$. 3D reconstruction showing 4 right pulmonary veins; 1 - right superior pulmonary vein; 2 - middle pulmonary vein; 3 - upper segment of the lower lobe vein; 4 - basilar segment vein;. E, F. CT scan showing a unique left vein; G. 3D reconstruction of a unique left vein; H. CT scan showing 3 left pulmonary veins; I. 3D reconstruction showing 3 left pulmonary veins; 1 - culminal vein; 2 - lingular vein; 3 - left lower lobe vein. 
2 as above (1\%) (Fig. 2D). Three (3\%) patients had a common right trunk. On the left side: the most common pattern was 2 veins, superior and inferior (89\%). However, we observed in one patient a lingular vein which drained in the left inferior vein (1\%). The most frequent anatomical variation was the existence of a single PV, occurring in $8(8 \%)$ cases (Fig. 2E, F, G). In 2 patients there were 3 PVs with 1 culminal vein, 1 lingular vein and 1 left inferior PV (2\%) (Fig. 2H, I).

\section{Implication in VATS lobectomy}

Among the 100 patients we included with the intention to perform a VATS lobectomy after multidisciplinary concertation, the surgical procedures performed were: $65(65 \%)$ lobectomies, $5(5 \%)$ pneumonectomies, $3(3 \%)$ bilobectomies, 8 (8\%) segmentectomies, 15 (15\%) wedge-resections after a negative frozen section and $4(4 \%)$ others procedures. Surgical conversion occurred in $21(21 \%)$ cases; it was due in $8(8 \%)$ cases to an extension of the surgical procedure towards a bilobectomy or a pneumonectomy, in $6(6 \%)$ patients to a difficulty in exposing the vascular elements within the fissure, a failure to conduce or maintain an unipulmonary ventilation in $5(5 \%)$ cases, and an arterial wound in $2(2 \%)$ cases. We didn't experience a pulmonary venous lesion $(0 \%)$ or a post-operative lung infarction $(0 \%)$. Among the 36 anatomical variations described radiologically in the number of pulmonary vein, 22 surgical procedures were concerned (lobar resections).

All these 22 variations were detected preoperatively and confirmed intraoperatively, without any pulmonary venous anatomical difference.

\section{DISCUSSION}

The anatomical limit of veno-atrial junction is differently defined between authors in the medical literature. For Jongbloed et al. [5, 6], limits of the left atrium on the CT-scan are extrapolated in an elliptical shape in the axial oblique cut in which the ostia of $\mathrm{PV}$ s are detectable.

Lacomis et al. [7] believe that the venous ostium is situated in the reflection of the pericardium; however, for Marom et al. [10], there is no exact definition, and anatomical limits of this junction are subjective. Moreover, the venous ostium is anatomically defined as the orifice of the vessel at the veno-atrial junction. This definition is ambiguous because of the conical shape of the vein entrance in the atrium. Table 1 shows the different venous anatomical variations found in the medical literature. Most of these studies are performed in the field of interventional cardiology for atrial fibrillation, using CT scan, ultrasound imagery or both. Nevertheless, the $\mathrm{CT}$ scan allows a better anatomical examination of the PVs and their variations compared to ultrasound imagery [5]. Moreover, the CT scan is included in the preoperative morphological assessment for lung cancer, and is more reachable during preoperative course for lung cancer than magnetic resonance imagery.

Our study confirms that the most common venous anatomical pattern is the presence of $2 \mathrm{PV}$ s on the right side, as on the left side, with a middle lobe vein draining in the right superior PV. The most commonly found variation is the single venous common trunk on the left side and three separate veins on the right side. The prevalence of a pulmonary venous common trunk is reported to vary from $10 \%$ to $79 \%$ on the left side and from $0 \%$ to $31 \%$ on the right side, with the higher variation rate in the study of Jongbloed et al. [5]. Also, in the study of Jongbloed et al. [5], including 42 patients, the frequency of a supernumerary vein was $32 \%$. However, Marom et al. [10] reported $14 \%$ of left venous common trunk and $28 \%$ of supernumerary veins ( 3 to 5 veins) on the right side, in over 205 patients with CT scan analysis. Schwartzman et al. [16] found a mean of $11.6 \%$ left venous common trunk and a mean of $22.2 \%$ right supernumerary PVs over $117 \mathrm{CT}$ scan. Our results confirm these findings. We estimated the prevalence of a venous anatomical variation in $36 \%$ patients in our study, including $11 \%$ of left venous common trunk and $17 \%$ of right supernumerary PVs. The other pulmonary venous anatomical variations described in the literature are less common and are mainly case reports, especially pertaining to the right superior pulmonary vein located posteriorly to the intermediate bronchus or a PV draining in the caval, portal or left brachio-cephalic systems [1]. The clinical implication of these anatomical variations regards pulmonary resections especially under videothoracoscopy in which the operator may be confronted to non-negligible disadvantages: reduction of the operating field or difficult vascular exposure, less understanding of the hilum anatomy, and lack of understanding of the path of the pulmonary veins, compared to open surgery. Knowing these anatomical variations and a precise examination of the preoperative CT scan is useful for all thoracic surgeons in order to perform a safe and precise surgical procedure $[1,18]$. Indeed, this preoperative assessment allowed us to avoid a venous wound or a post-operative lung infarction in our study. Moreover, a preoperatively unidentified pulmonary venous anatomical variation may remain 
Table 1. Pulmonary venous variations found in the literature

\begin{tabular}{|c|c|c|c|c|c|c|c|}
\hline Author & Year & Analysis & Variations & 3 RPV (\%) & LCT (\%) & $\begin{array}{c}\text { MPV } \\
\text { drained in } \\
\text { RIPV }(\%)\end{array}$ & Other \\
\hline Gokhan [4] & 2008 & CT-scan & & 22 & 29 & & \\
\hline Shunsuke [17] & 2010 & CT-scan & $5.8 \%(5 / 86)$ & & 20 & 20 & $\begin{array}{c}1 \text { lingular vein drained } \\
\text { in the LIPV, } 1 \text { posterior RPV, } \\
1 \text { LSPV drained in the left } \\
\text { inominate vein }\end{array}$ \\
\hline Rajeshwari [14] & 2012 & $\begin{array}{l}\text { Cadaveric } \\
\text { dissetion }\end{array}$ & $46.03 \%$ & 26.9 & & 11.53 & \\
\hline Shunsuke [17] & 2009 & CT-scan & & & & & $\begin{array}{l}1 \text { RSPV posterior to the } \\
\text { bronchus intermedius }\end{array}$ \\
\hline Marom [10] & 2004 & CT-scan & $\begin{array}{l}\text { Right: } 32 \% \\
\text { Left: } 14 \%\end{array}$ & 26 & 14 & & \\
\hline Sasaki [15] & 2006 & CT-scan & & & & & RSPV drained in SVC \\
\hline Yamashita [21] & 1978 & & & & & 4.8 & \\
\hline Lovesh [9] & 2012 & $\begin{array}{c}\text { Cadaveric } \\
\text { dissetion }\end{array}$ & $44.8 \%(13 / 29)$ & 10.3 & 17.2 & & $\begin{array}{l}1 \text { RCT: } 3.4 \% \\
4 \text { RPV: } 3.4 \% \\
3 \text { LPV: } 3.4 \%\end{array}$ \\
\hline Lickfett [8] & 2005 & MRI & & 8 & 12 & & \\
\hline Micochova [11] & 2005 & MRI & & 23 & & & \\
\hline Jongbloed $[5,6]$ & 2005 & CT-scan & & & 79 & & $\begin{array}{c}1 \mathrm{RCT}: 31 \% \\
\text { Additional RPV: } 29 \%\end{array}$ \\
\hline Schwartzman [16] & 2003 & CT-scan & & 22.2 & 11.6 & & \\
\hline Current study & 2016 & CT-scan & $36 \%(36 / 100)$ & 16 & 8 & 4 & $\begin{array}{l}1 \text { RCT: } 3 \% \\
4 \text { RPV: } 1 \% \\
3 \text { LPV: } 2 \%\end{array}$ \\
\hline
\end{tabular}

CT — computed tomography; LCT — left common trunk; LIPV — left inferior pulmonary vein; LPV — left pulmonary vein; LSPV — left superior pulmonary vein; MPV — middle pulmonary vein; MRI — magnetic resonance imagery; RCT — right common trunk; RPV — right pulmonary vein; RSPV — right superior pulmonary vein; SVC — superior vena cava

unknown during the intervention, as a result of a bad hilar exposure, and/or minimal dissection $[13,15,18]$. Failure in the identification of the pulmonary venous variations may lead to an intraoperative venous lesion of a supernumerary vein, or a venous ligature of a nonresected segment or lobe, when the ligation interests a common trunk. We identified venous anatomical variations at risk for venous lesions: a supernumerary vein for the superior segment of the right inferior lobe can be wounded during a right inferior lobectomy if not identified before. Also, a separated vein for the lingular segment can be wounded during a left upper lobectomy. We also identified anatomical variations at risk for venous ligature of a non-resected segment or lobe that might cause infarction and necrosis of the remaining lung parenchyma: when there is a middle lobe vein draining in the inferior pulmonary vein, if it hasn't been identified before or during surgery, infarction and necrosis of the middle lobe might occur during right lower lobectomy after ligation of the inferior PV. The same might occur on the opposite side when there is a pulmonary inferior vein receiving a venous branch from the lingula. Minamoto et al. [12] report a left inferior $\mathrm{PV}$ receiving venous branches from the lingula and one basal segment. Preoperative CT scan identification of this varying pattern enhanced the understanding of the anatomy and allowed a precise dissection and preservation of the lingular vein [12].

The existence of a pulmonary venous common trunk, on both sides, is also considered as a risky situation. Nakamura et al. [13] reported a case of closing a left common trunk during a left upper lobectomy. This anatomical variation has not been detected in preoperative $\mathrm{CT}$ scan examination but found per-operatively after dividing the common trunk instead of the left superior vein, imposing the author to perform a venous anastomosis between the left inferior PV and left atrium [13]. Preoperative assessment of these variations appears relevant and when 
there is still doubt after CT scan analysis, we recommend a thorough intraoperative examination of the origin, path and destination of each pulmonary vein. If needed, this may lead to an intrapericardial dissection of the venous vessels, in order to fully understand the pulmonary venous anatomy and perform a safe VATS procedure.

\section{CONCLUSIONS}

In conclusion, we highlight the pulmonary venous anatomical variations at risk for vascular complications, especially under VATS lobectomy procedure. Anatomical variations exist and pre-operative study of PVs using $C T$ scan is very important in preventing unnecessary extension of pulmonary resections or iatrogenic complications such as per-operative bleeding, or parenchyma infarction/necrosis in a lung cancer surgery.

The existence of a venous common trunk or supernumerary veins should lead to a thorough dissection of the pulmonary hilum to enhance surgical management of lung cancer with these venous anatomical variations.

\section{REFERENCES}

1. Asai $K$, Urabe N, Yajima K, et al. Right upper lobe venous drainage posterior to the bronchus intermedius: preoperative identification by computed tomography. Ann Thorac Surg. 2005; 79(6): 1866-1871, doi: 10.1016/j.athoracsur.2004.12.014, indexed in Pubmed: 15919274.

2. Douglas $\mathrm{YL}$, Jongbloed MRM, Deruiter MC, et al. Normal and abnormal development of pulmonary veins: state of the art and correlation with clinical entities. Int J Cardiol. 2011; 147(1): 13-24, doi: 10.1016/j.ijcard.2010.07.004, indexed in Pubmed: 20674049.

3. Fukuhara K, Akashi A, Nakane S, et al. Preoperative assessment of the pulmonary artery by three-dimensional computed tomography before video-assisted thoracic surgery lobectomy. Eur J Cardiothorac Surg. 2008; 34(4): 875-877, doi: 10.1016/j.ejcts.2008.07.014, indexed in Pubmed: 18703345.

4. Gokhan A, Elvan D, Adnan A, et al. Right top pulmonary vein: evaluation with 64 section multidetector computed tomography. Eur J Radiol. 2008; 67(2): 300-303, doi: 10.1016/j. ejrad.2007.07.005, indexed in Pubmed: 17703907.

5. Jongbloed MRM, Bax JJ, Lamb HJ, et al. Multislice computed tomography versus intracardiac echocardiography to evaluate the pulmonary veins before radiofrequency catheter ablation of atrial fibrillation: a head-to-head comparison. J Am Coll Cardiol. 2005; 45(3): 343-350, doi: 10.1016/j. jacc.2004.10.040, indexed in Pubmed: 15680710.

6. Jongbloed MRM, Dirksen MS, Bax JJ, et al. Atrial fibrillation: multi-detector row CT of pulmonary vein anatomy prior to radiofrequency catheter ablation--initial experience. Radiology. 2005; 234(3): 702-709, doi: 10.1148/radiol.2343031047, indexed in Pubmed: 15665218.

7. Lacomis JM, Wigginton W, Fuhrman C, et al. Multi-detector row $\mathrm{CT}$ of the left atrium and pulmonary veins before radiofrequency catheter ablation for atrial fibrillation. Radiograph- ics. 2003; 23 Spec No: S35-48; discussion S48, doi: 10.1148/ rg.23si035508, indexed in Pubmed: 14557500.

8. Lickfett L, Dickfeld T, Kato R, et al. Changes of pulmonary vein orifice size and location throughout the cardiac cycle: dynamic analysis using magnetic resonance cine imaging. J Cardiovasc Electrophysiol. 2005; 16(6): 582-588, doi: 10.1046/j.1540-8167.2005.40724.x, indexed in Pubmed: 15946353.

9. Lovesh S, Neha G, Gargi S, et al. Variation in Number and Drainage Pattern of Pulmonary Veins Draining into the Left Atrium. J Anat Soc India. 2012; 61(1): 5-8, doi: 10.1016/ s0003-2778(12)80003-7.

10. Marom EM, Herndon JE, Kim YH, et al. Variations in pulmonary venous drainage to the left atrium: implications for radiofrequency ablation. Radiology. 2004; 230(3): 824-829, doi: 10.1148/radiol.2303030315, indexed in Pubmed: 14739316.

11. Mlcochová H, Tintera J, Porod V, et al. Magnetic resonance angiography of pulmonary veins: implications for catheter ablation of atrial fibrillation. Pacing Clin Electrophysiol. 2005; 28(10): 1073-1080, doi: 10.1111/j.15408159.2005.00228.x, indexed in Pubmed: 16221266.

12. Minamoto K, Misao T, Takashima S, et al. Successful thoracoscopic lobectomy for lung cancer in a patient with anatomic variation of the left inferior pulmonary vein. Acta Med Okayama. 2007; 61(2): 103-106, indexed in Pubmed: 17471311.

13. Nakamura T, Koide $M$, Nakamura $H$, et al. The common trunk of the left pulmonary vein injured incidentally during lung cancer surgery. Ann Thorac Surg. 2009; 87(3): 954-955, doi: 10.1016/j.athoracsur.2008.07.054, indexed in Pubmed: 19231436.

14. Rajeshwari MS, Ranganath P. Variations in draining patterns of right pulmonary veins at the hilum and an anatomical classification. ISRN Pulmonology. 2012; 2012: 1-4, doi: 10.5402/2012/786549.

15. Sasaki H, Naka N, Kitahara N, et al. Right partial anomalous pulmonary venous connection found during lobectomy for coexisting lung cancer and tuberculosis: report of a case. Clin Lung Cancer. 2006; 7(5): 350-352, indexed in Pubmed: 16640808.

16. Schwartzman D, Lacomis J, Wigginton WG. Characterization of left atrium and distal pulmonary vein morphology using multidimensional computed tomography. J Am Coll Cardiol. 2003; 41(8): 1349-1357, indexed in Pubmed: 12706931.

17. Shunsuke $E$, Hiroyoshi T, Tomoyuki N, et al. A dangerous venous variation in thoracoscopic right lower lobectomy. Ann Thorac Surg. 2009; 87(2): e9-ee10, doi: 10.1016/j. athoracsur.2008.08.051, indexed in Pubmed: 19161734.

18. Shunsuke Y, Atsushi S, Yoshimasa I, et al. Importance of preoperative assessment of pulmonary venous anomaly for safe video-assisted lobectomy. Interact Cardiovasc Thorac Surg. 2010; 10(6): 851-854, doi: 10.1510/icvts.2009.221804, indexed in Pubmed: 20332219.

19. Webb S, Kanani M, Anderson RH, et al. Development of the human pulmonary vein and its incorporation in the morphologically left atrium. Cardiol Young. 2001; 11(6): 632-642, indexed in Pubmed: 11813915.

20. William L. Embryologie humaine. 2nd Ed. De Boeck 2003: p. 548.

21. Yamashita H. Roentgenologic anatomy of the lung. IgakuShoin, Tokyo. 1978; 1978: 70-107. 